\title{
MODELOCKING USING MIRRORS WITH INTENSITY DEPENDENT REFLECTION COEFFICIENTS
}

\author{
J.R.M. BARR \\ Department of Physics, University of Southampton, Southampton S09 5 NII, UK
}

Received 3 October 1988

\begin{abstract}
The steady state modelocking of a laser incorporating a mirror with an intensity dependent reflection coefficient is analysed. The pulse length is determined by the balance between pulse stretching due to gain narrowing and pulse shortening due to the nonlinear mirror. The final pulse length can approach the limit set by the gain bandwidth of the active medium.
\end{abstract}

\section{Introduction}

Recently it has been demonstrated that second harmonic generation can be used to provide a mirror with an intensity dependent reflection coefficient $[1,2]$. In particular the reflectivity can increase with intensity so that when used intracavity the laser will tend to become passively modelocked. Using such a scheme pulses as short as $\sim 100$ ps at the second harmonic wavelength have been generated after only $\sim 16$ cavity round trips. However it is clear that the lower limit on pulse length should ultimately be set by the gain bandwidth of the active medium or by group velocity dispersion in the nonlinear crystal, this limit has not been reached so far. An analysis of this method of modelocking, based on an extension of the well tested theory of active modelocking in solid state lasers [3], is presented in this paper. It demonstrates that the steady state pulse length is determined by a balance between pulse shortening by the nonlinear mirror and pulse stretching through gain narrowing by the active medium and that pulse durations approaching those set by the limiting bandwidth can be achieved in principle.

\section{Theory}

The nonlinear mirror consists of a second harmonic crystal separated by an adjustable distance from a mirror. The mirror has a different intensity reflection coefficient for the harmonic, $R_{2 \omega}$, than for the fundamental $R_{t,}\left(R_{2(t)}>R_{i,}\right)$. If the mirror-crystal separation is adjusted to give the correct phase difference between the fundamental and harmonic, via the dispersion in air and the dispersion associated with the mirror reflectivity, then the fundamental can be amplified by the second harmonic [4]. This increases the effective reflectivity over that of the mirror alone. The effect of the nonlinear mirror is similar to a saturable absorber but with a much faster time constant. The full expression for the nonlinear mirror intensity reflectivity, $R_{\mathrm{N} 1}$, is derived in ref. [1] for a conversion efficiency of $\eta$ from the fundamental to the harmonic on the first pass through the doubling crystal and is

$$
\begin{aligned}
R_{\mathrm{NL}} & =B\left\{1-\tanh ^{2}[\sqrt{B} \operatorname{arctanh}(\sqrt{\eta})\right. \\
& \left.\left.-\operatorname{arctanh}\left(\sqrt{\eta R_{2 \sigma \nu} / B}\right)\right]\right\},
\end{aligned}
$$

where

$B=\eta R_{2 \omega}+(1-\eta) R_{\omega}$.

The normalized reflectivity $R_{\mathrm{NL}} / R_{\omega}$ is shown in fig. 1 (a) while fig. 1 (b) shows the normalised reflectivity plotted on a logarithmic scale. The curves nearly have an exponential dependence on $\eta$ so that the following approximation can be made

$R_{\mathrm{NL}}=R_{\omega} \exp (\sigma \eta)$,

which agrees within $5 \%$ of the exact curve calculated using eq. (1) for $R_{2 \omega}=1$. The parameter $\sigma$ is a func- 

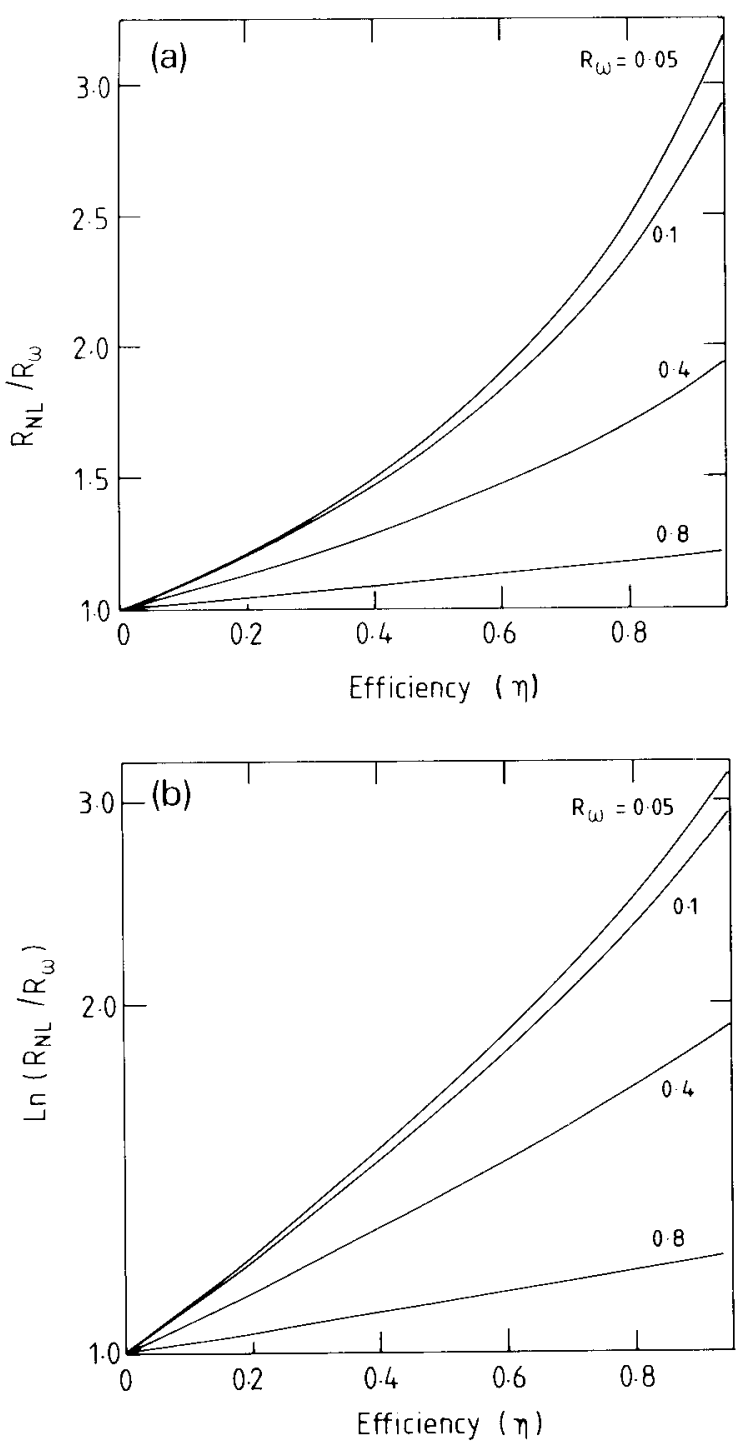

Fig. 1. (a) Normalised intensity dependent reflectivity of the nonlinear mirror-plotted for a number of values of the fundamental mirror reflectivity $R_{\omega,}$. The reflectivity for the second harmonic is $R_{20}=1$. (b) The same graph as figure 1 (a) but with the normalised reflectivity plotted on a logarithmic scale.

tion of $R_{\ell,}$ and values are given in table 1 . This approximation will be used to derive an analytical form for the steady state pulse length.

The cavity that will be analysed is shown in fig. 2 . The light travels clockwise round the resonator, first passing through the modulator, then the active medium, then the modulator and finally the nonlinear mirror. The amplitude modulator is included for two reasons. Firstly it enables the well known gaussian pulse solutions to be obtained if the second harmonic conversion is switched off [3]. Secondly the laser operation will be stabilised by the inclusion of the modulator. Similar effects have been observed in active/passive modelocking of solid state lasers where the passive medium is a saturable dye [5]. An additional practical advantage is that the of voltage, used to drive the modulator, can be used to synchronise the laser with other equipment.

The passage of the light pulse, described by its electric field amplitude $f(t)$, through the gain medium and modulator in the time domain is

$f_{2}(t)=m(t)\left[g_{2}(t) \otimes f_{1}(t)\right]$.

Here $g_{2}(t)$ is the Fourier transform of the double pass frequency dependent gain and the amplitude transmission of the modulator in double pass is described by $m(t)$. It has been assumed that the ordering of the effects of the modulator and gain medium does not significantly modify $f_{2}(t)$. This is a realistic assumption since the magnitude of the modulator will be shown not change the steady state pulse length significantly. The symbol $\otimes$ represents convolution defined using the Fourier transform pair

$$
\begin{aligned}
& f(\omega)=\frac{1}{2 \pi} \int f(t) \exp (-\mathrm{i} \omega t) \mathrm{d} t, \\
& f(t)=\int f(\omega) \exp (\mathrm{i} \omega t) \mathrm{d} \omega,
\end{aligned}
$$

to be

$g_{2} \otimes f_{1}(t)=\frac{1}{2 \pi} \int g_{2}\left(t^{\prime}\right) f_{1}\left(t-t^{\prime}\right) \mathrm{d} t^{\prime}$.

Next, the pulse $f_{2}(t)$ is passed through the non-linear mirror. A pulse compression results since the nonlinear reflectivity is less in the wings of the pulse than at the peak. The conversion efficiency for each point on the pulse profile is found from the analysis in ref. [1] or [4] to be

$$
\begin{aligned}
\eta(t) & =\tanh ^{2}(K f(t)) \\
= & \tanh ^{2}\left\{\left[f(t) / f_{\text {max }}\right] \operatorname{arctanh}\left(\sqrt{\eta_{0}}\right)\right\} \\
\approx & \eta_{0} f^{2}(t) / f_{\text {max }}^{2},
\end{aligned}
$$

where $K$ is a constant which includes the crystal properties and length, $\eta_{0}$ is the conversion efficiency at the peak of the pulse, $f_{\max }$. The last part of eq. (4) 


\begin{tabular}{|c|c|c|c|c|c|c|}
\hline \multirow[t]{2}{*}{$R_{t, 1}$} & \multirow[t]{2}{*}{$\sigma$} & \multirow[t]{2}{*}{$\eta$} & \multirow{2}{*}{$\begin{array}{l}\text { Gain } \\
G \\
\text { (a) }\end{array}$} & \multicolumn{3}{|c|}{ Pulse length (ps) } \\
\hline & & & & $\begin{array}{l}\text { active } \\
\text { modelocking } \\
\text { (b) }\end{array}$ & $\begin{array}{l}\text { approximate } \\
\text { (c) }\end{array}$ & $\begin{array}{l}\text { exact } \\
\text { (d) }\end{array}$ \\
\hline 0.8 & 0.21 & 0.1 & 1.22 & 72.5 & 13.8 & 21.3 \\
\hline 0.3 & 0.85 & 0.1 & 3.06 & 111.3 & 16.7 & 25.8 \\
\hline 0.2 & 1.0 & 0.1 & 4.52 & 119.9 & 18.0 & 27.2 \\
\hline 0.2 & 1.0 & 0.2 & 4.09 & 117.8 & 12.8 & 18.4 \\
\hline 0.2 & 1.0 & 0.3 & 3.70 & 115.7 & 10.5 & 14.7 \\
\hline 0.2 & 1.0 & 0.5 & 3.03 & 111.0 & 8.1 & 10.6 \\
\hline
\end{tabular}

a) Calculated from $\exp \left(g_{2}\right) \sqrt{R_{r o}} \exp \left(\sigma \eta_{0} / 2\right)=1$ and $G=\exp \left(2 g_{2}\right)$.

b ' Calculated assuming $\eta=0$ from eq. (10), $A=1$, and $f_{\mathrm{m}}=100 \mathrm{MHz}$.

"Calculated using eq. (14).

d) Calculated using an iterative method from eq. (9).

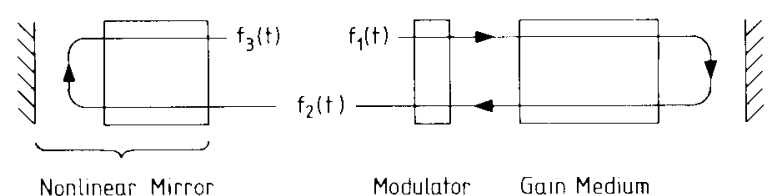

Fig. 2. The model laser cavity. $f_{1}(t)$ is the starting electric field pulse profile while $f_{2}(t)$ is the pulse profile after a double pass of the gain medium and amplitude modulator. Passage of $f_{2}(t)$ through the nonlinear mirror results in $f_{3}(t)$. For a steady state solution $f_{3}(t)=f_{1}(t)$.

is valid if depletion of the fundamental may be neglected. The reflected pulse $f_{3}(t)$ is found from eq. (1) to be

$f_{3}(t)=\sqrt{R_{\mathrm{NL}}(t)} f_{2}(t)$,

remembering that $R_{\mathrm{NL}}$ is the intensity reflectivity of the nonlinear mirror. The time dependence arises through the conversion efficiency from eq. (4). The final expression for the pulse evolution through a single round trip of the cavity is

$f_{3}(t)=\sqrt{R_{\mathrm{NL}}(t)} m(t)\left[g_{2} \otimes f_{1}(t)\right]$,

and for self consistency the condition $f_{3}(t)=f_{1}(t)$ is applied. This condition implicitly assumes that the round trip time, taking into account the dispersion in the amplifier and other components, is equal to the modulation frequency. Notice that the inclusion of the nonlinear mirror means that the self consistent profile is no longer gaussian. This complicates the analysis, but it will be shown that the peak of the pulse can, under certain conditions, be approximated by a gaussian. In this way an analytical estimate of the steady state pulse length can be obtained. To do this the modulator transmission $m(t)$ and the gain, $g_{2}(\omega)$, will be assumed to be gaussian in shape. The modulator transmission is written as

$$
\begin{aligned}
& m(t)=\exp \left[-\Delta\left(1-\cos \omega_{\mathrm{m}} t\right)\right] \\
& \quad \approx \exp \left(-\Delta \omega_{\mathrm{m}}^{2} t^{2} / 2\right)
\end{aligned}
$$

where $\Delta$ is the double pass modulation index. The gain is approximated by a gaussian profile as

$$
\begin{array}{r}
g_{2}(\omega)=\exp \left[\frac{g_{2}}{1+2 \mathrm{i}\left(\omega-\omega_{\mathrm{a}}\right) / \delta \omega_{\mathrm{a}}}\right] \approx \exp \left(g_{2}\right) \\
\quad \times \exp \left[-2 g_{2} \mathrm{i}\left(\frac{\omega-\omega_{\mathrm{a}}}{\delta \omega_{\mathrm{a}}}\right)-4 g_{2}\left(\frac{\omega-\omega_{\mathrm{a}}}{\delta \omega_{\mathrm{a}}}\right)\right] .
\end{array}
$$

The function $g_{2}(t)$ is calculated using the appropriate Fourier transform

$$
\begin{aligned}
& g_{2}(t)=\int g_{2}(\omega) \exp (\mathrm{i} \omega t) \mathrm{d} \omega=(\pi / \alpha)^{1 / 2} \\
& \quad \times \exp \left(g_{2}\right) \exp \left[-(t-\beta)^{2} / 4 \alpha\right] \exp \left(\mathrm{i} \omega_{\mathrm{a}} t\right),
\end{aligned}
$$

where $\alpha=4 g_{2} / \delta \omega_{\mathrm{a}}^{2}$ and $\beta=2 g_{2} / \delta \omega_{\mathrm{a}}$.

Assuming a gaussian input pulse with no frequency chirp centred at the peak of the gain profile

$f_{1}(t)=f_{0} \exp \left(-\gamma t^{2}\right) \exp \left(\mathrm{i} \omega_{\mathrm{a}} t\right)$

then from eqs. (3), (6) and (7), $f_{2}(t)$ is 


$$
\begin{aligned}
& f_{2}(t)=\frac{f_{0} \exp \left(g_{2}\right)}{(1+4 \alpha y)^{1 / 2}} \exp \left(\mathrm{i} \omega_{\mathrm{a}} t\right) \\
& \times \exp \left[-\left(\frac{y}{1+4 \alpha y}+\frac{A \omega_{\mathrm{m}}^{2}}{2}\right)(t-\beta)^{2}\right],
\end{aligned}
$$

and the reflected pulse from the nonlinear mirror is $f_{3}(t)=\sqrt{R_{\mathrm{NL}}(t)} f_{2}(t)$.

If the conversion efficiency, $\eta=0$, then this reduces to

$$
\begin{aligned}
& f_{3}(t)=\frac{\sqrt{R_{(}}, f_{0} \exp \left(g_{2}\right)}{(1+4 \alpha y)^{1 / 2}} \exp \left(\mathrm{i} \omega_{\mathrm{a}} t\right) \\
& \times \exp \left[-\left(\frac{y}{1+4 \alpha y}+\frac{\Delta \omega_{\mathrm{m}}^{2}}{2}\right)(t-\beta)^{2}\right],
\end{aligned}
$$

which, together with the self consistent condition leads to the well known solutions for the equilibrium value of $y$ and the full width at half maximum of the intensity $\tau_{\mathrm{m}}$,

$y_{\mathrm{m}}=\left(\Delta \omega_{\mathrm{m}}^{2} / 8 \alpha\right)^{1 / 2}$

$\tau_{\mathrm{m}}=\left[(2 \ln 2) / \pi^{2}\right]^{1 / 2}\left(2 g_{2} / \Delta\right)^{1 / 4}\left(1 / f_{\mathrm{m}} f_{\mathrm{a}}\right)^{1 / 2}$.

Using the approximation of eq. (2) in eq. (9) for low conversion efficiency, $\eta_{0}<0.2$ yields

$f_{3}(t)=\sqrt{R_{t,}} \exp \left(\frac{\sigma \eta_{0}}{2} \frac{f_{2}^{2}(t)}{f_{2}^{2}(0)}\right) f_{2}(t)$.

Near the peak of the pulse the exponential in eq. (11) may be expanded and retaining only quadratic terms

$\frac{f_{2}^{2}(t)}{f \frac{2}{2}(0)} \approx\left[1-2\left(\frac{y}{1+4 \alpha \gamma}+\frac{\Delta \omega_{\mathrm{m}}^{2}}{2}\right) t^{2}+\ldots\right]$,

which together with $(8)$ gives

$$
\begin{aligned}
& f_{3}(t)=\left[R_{o} /(1+4 \alpha y)\right]^{1 / 2} f_{0} \exp \left(g_{2}\right) \\
& \quad \times \exp \left(\mathrm{i} \omega_{\mathrm{a}} t\right) \exp \left(\sigma \eta_{0} / 2\right) \\
& \quad \times \exp \left[-\left(1+\sigma \eta_{0}\right)\left(\frac{y}{1+4 y \alpha}+\frac{\Delta \omega_{\mathrm{m}}^{2}}{2}\right) t^{2}\right] .
\end{aligned}
$$

The application of self consistency gives $y$ as

$y=\frac{\sigma \eta_{0}}{8 \alpha\left(1+\sigma \eta_{0}\right)} \pm \frac{1}{2}\left[\left(\frac{\sigma \eta_{0}}{4 \alpha\left(1+\sigma \eta_{0}\right.}\right)^{2}+\frac{\Delta \omega_{\mathrm{m}}^{2}}{2 \alpha}\right]^{1 / 2}$.
Notice that if $\eta_{0}=0$ then this reduces to eq. (10). For most values of $\eta_{0}$ the final term under these square root sign may be neglected, so the steady state values of $y$ are

$y_{\eta}=\sigma \eta_{0} / 4 \alpha\left(1+\sigma \eta_{0}\right)$, or $y_{\eta}=0$.

Thus there is the possibility of two states of the laser. Either there is a constant output which corresponds to $y=0$ or there is a modelocked output with fwhm intensity width $\tau_{\eta}$,

$\tau_{\eta}=\frac{2 \sqrt{2 \ln (2)}}{\pi}\left(\frac{g_{2}\left(1+\sigma \eta_{0}\right)}{\sigma \eta_{0}}\right)^{1 / 2} \frac{1}{f_{\mathrm{a}}}$.

The question of which state a laser will tend to operate in is open and depends on the detailed laser dynamics. However, the inclusion of a modelocker does reduce the number of states available to the laser from two to one since only the positive sign can be taken in eq. (13) [3]. Note that eq. (13) can be written in the form

$y=y_{\eta} / 2 \pm\left[\left(y_{\eta} / 2\right)^{2}+y_{\mathrm{m}}^{2}\right]^{1 / 2}$,

where the negative sign may only be taken if $y_{\mathrm{m}}=0$. In this case $y_{\eta}$ is the value of $y$ if there is no active modelocking ( $y_{\mathrm{m}}=0$ ) while $y_{\mathrm{m}}$ is the value of $y$ if the nonlinear mirror makes no contribution $\left(y_{\eta}=0\right)$.

\section{Discussion}

A number of values of steady state pulse lengths have been calculated using the approximations of eq. (14) and an iterative numerical method based on eq. (9), and are displayed in table 1 . The laser bandwidth, $f_{\mathrm{a}}$, corresponded to $\mathrm{Nd}$ : YAG, $f_{\mathrm{a}}=120 \mathrm{GHz}$. The modulator frequency, $f_{\mathrm{m}}$, was $100 \mathrm{MHz}$ while the modulation depth, $A$, was 1 . The modulator had very little effect on the resultant pulse length if $f_{\mathrm{m}}$ and $\Delta$ were varied over the range of presently achievable values. For example, variation of $A$ over the range 0.1 to 2 varied the pulse length calculated using eq. (9) by less than $0.1 \%$. Fig. 3 illustrates a typical steady state pulse profile together with a gaussian pulse with the same fwhm. Significant differences only occur in the wings of the pulse. It is clear from table 1 that the approximate pulse length is in agreement with the exact pulse length but is systematically smaller by about $30 \%$. The accuracy of 


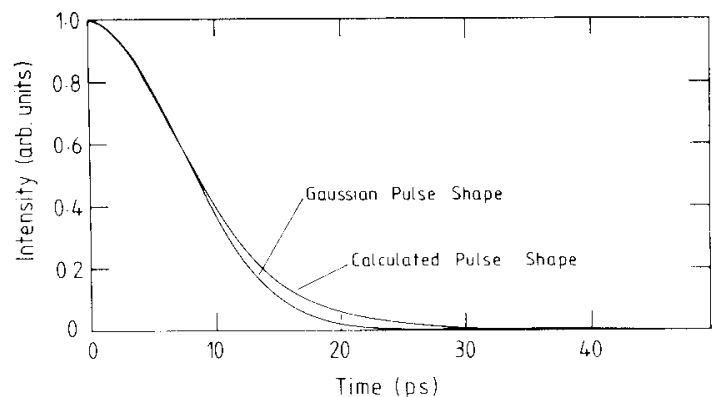

Fig. 3. The stcady state profile calculated numerically for $R_{t,}=0.2$, $\eta=0.2$. Also shown is a gaussian pulse with the same fwhm. The agreement over much of the profile justifies the approximation of eqs. (2) and (12).

the numerical technique was checked by setting the conversion efficiency to zero and generating the standard active modelocking pulse durations which may be calculated using eq. (10). Typically the numerical method and eq. (10) differed by $1.5 \%$ or less. An example of the pulse length reduction that could be achieved by the inclusion of a nonlinear mirror may be found considering a modelocked $\mathrm{cw} \mathrm{Nd}$ :YAG laser. Active modelocking of such a system with $R_{\omega}=80 \%$ and $\Delta=1$ yields a pulse length of 72.5 ps which with the inclusion of a nonlinear mirror can be reduced to $21.3 \mathrm{ps}$, assuming a peak steady state conversion efficiency of $\eta_{0}=0.1$.

In deriving the steady state pulse length the question of whether the laser will reach this condition has not been answered. As the pulse forms the conversion efficiency increases because the peak intensity increases. The pulse energy should increase slightly over the $\eta=0$ case due to the reduction in threshold [2], and this may alter the approach to steady state through the relaxation oscillations. An additional effect which could modify the laser properties is due to the transverse mode profile. The conversion efficiency varies spatially as does the reflectivity of the non-linear mirror. The stability of such a laser is at present under study.
If the bandwidth of the active medium is sufficiently large then group velocity differences between the fundamental and harmonic in the doubling crystal will limit the achievable pulse length. This will also introduce a time delay in the passage through the nonlinear mirror [6,7] and will complicate the arguments used in this paper.

The results indicate that this method can be used to modelock lasers with a wide range of properties provided that a sufficiently high peak conversion can be obtained. In particular it may have benefits in the case of novel tunable solid state lasers which can have very large bandwidths.

\section{Conclusion}

A simple analysis of modelocking using a mirror with an intensity dependent reflectivity coefficient has been presented. The pulse formation mechanisms represents a balance between pulse stretching in the gain medium and pulse shortening in the nonlinear mirror. Using the fact that in steady state these processes are balanced, the resulting pulse length may be calculated numerically. A simple analytic solution was obtained with a wide range of validity. This modelocking scheme has the benefit that it may use much of the available bandwidth the active medium.

\section{References}

[1] K.A. Stankov, Appl. Phys. B45 (1988) 191.

[2] K.A. Stankov and J. Jethwa, Optics Comm, 66 (1988) 41.

[3] D.A. Kuizenga and A.E. Siegman, J. Quant. Electron. QE-6 (1970) 694.

[4]J.A. Armstrong, N. Bloembergen, J. Ducuing and P.S. Pershan, Phys. Rev, 127 (1962) 1918.

[5] H.P. Kortz. IEEE J. Quant. Electron. QE-19 (1983) 578.

[6] Y. Ishida and T. Yajima, Optics Comm. 62 (1987) 197.

[7] W.H. Glenn, IEEE J. Quant. Electron. QE-5 ( 1969) 284. 